\title{
Color Image Indexing Using Mathematical Morphology
}

\author{
Eugen Zaharescu
}

\begin{abstract}
A mathematical morphology based approach for color image indexing is explored in this paper. Morphological signatures are powerful descriptions of the image content in the framework of mathematical morphology. A morphological signature (either a pattern spectrum or a differential morphological profile) is defined as a series of morphological operations (namely openings and closings) considering a predefined pattern called structuring element. For image indexing it is considered a morphological feature extraction algorithm which includes more complex morphological operators: i.e. color gradient, homotopic skeleton, Hit-or-Miss transform. In the end, illustrative application examples of the presented approach on real acquired images are also provided.
\end{abstract}

\section{Introduction}

Morphological image analysis based on Mathematical Morphology (MM) theory uses the lattice theory, the set theory and Euclidian geometry to investigate the image spatial structures, the shape features of the image objects and the relationships between them ([6], [7], [8]). Based upon the MM theory, the precise detection of the object's pixels along with pertinent indexing features, proved to be more computationally efficient than other approaches (i.e. statistical methods concentrate on individual pixel values). But the extension of mathematical morphology for multivariate functions or multichannel images (e.g. color images) is a challenging approach. The definitions of the whole pyramid of the morphological operators starting from the basic ones (erosion

Key Words: Morphological signature, morphological feature extraction algorithm, color gradient, homotopic skeleton, Hit-or-Miss transform.

Received: November, 2013

Accepted: January, 2014 
and dilation) up to the next upper levels of derived operators (opening, closing, skeleton, top-hat, etc.) use a totally ordered complete lattice structure. This algebraic structure cannot be defined in a naturally or perceptually correct way onto the vector space of color images. The vector mathematical morphology theory have been analyzed in some fundamental papers ([1], [2], [3]). Several different orderings have been proposed for the vector space of color images:

- Marginal ordering uses the artificial point wise ordering (i.e. it orders color vectors component by component independently). In this case the disadvantage is that "false colors" (new color vector values that are not present in the input image) can be introduced in the processed image.

- Conditional ordering performs the ordering of color vectors by means of some marginal components selected sequentially according to different conditions (i.e. lexicographic ordering). The conditional ordering preserves the input color vectors and has been studied especially for HSV (Hue/Saturation/Value) representation of color images.

- Reduced ordering realizes the ordering of color vectors according to some scalars, computed from the components of each vector with respect to different measure criteria, typically distances or projections. The reduced ordering has been used to define morphological operators, in the framework of color morphology, by means of distances. It can be successfully used in filtering applications as well as the conditional ordering. $([9],[10])$

For the morphological operators we will use the classical functional definition from [5] and [6], where the morphological erosion $\left(\varepsilon_{g}(f)\right)$ and morphological dilation $\left(\delta_{g}(f)\right)$ are respectively defined as follows:

$\varepsilon_{g}(f)(x)=(f \ominus g)(x)=\inf \{f(x-y)-\breve{g}(y) \mid y \in S u p p(g)\}, \forall x \in S u p p(f) \subseteq \mathbb{R}^{n}$

$\delta_{g}(f)(x)=(f \oplus g)(x)=\sup \{f(x-y)+\breve{g}(y) \mid y \in S u p p(g)\}, \forall x \in S u p p(f) \subseteq \mathbb{R}^{n}$

where the grayscale image $f: E_{f} \subseteq \mathbb{R}^{n} \rightarrow \mathbb{R}$ and the structuring element or the structuring function $g: E_{g} \subseteq \mathbb{R}^{n} \rightarrow \mathbb{R}$ are semi-continuous functions and $\operatorname{Supp}(f)=E_{f}$ and $\operatorname{Supp}(g)=E_{g}$ are the definition domains for each function (in the following experiments $\operatorname{Supp}(f)$ and $\operatorname{Supp}(g)$ are Euclidian spaces, $E_{f}, E_{g} \subseteq \mathbb{R}^{2}$ or $E_{f}, E_{g} \subseteq \mathbb{Z}^{2}$, in case of an integer grid). Also $\breve{g}$ is the symmetric structuring function defined as follows: $\forall x \in \mathbb{R}^{n}, \breve{g}(x)=g(-x)$.

In case of 2-D binary images these basic morphological operators binary erosion $(\ominus)$ and binary dilation $(\oplus)$ are respectively rewritten more simplified:

$$
A \ominus B=\left\{(x, y) \mid B_{(x, y)} \subseteq A\right\}=\bigcap_{(u, v) \in B} A_{(-u,-v)}=\bigcap_{(u, v) \in B^{S}} A_{(u, v)}
$$




$$
A \oplus B=\left\{(x, y) \mid B_{x, y} \bigcap A \neq\{\phi\}\right\}=\bigcup_{(u, v) \in B} A_{(-u,-v)}=\bigcup_{(u, v) \in B^{S}} A_{u, v}
$$

where $A$ is a $2-D$ binary image and $B$ is a $2-D$ structuring element defined on an Euclidian space, $E \subseteq \mathbb{Z}^{2} . A_{(u, v)}$ and $B_{(x, y)}$ are the translations of $A$ and $B$ by vectors $(u, v)$ and $(x, y) . B^{S}$ denotes the symmetric structuring element $B$ :

$$
B^{S}=\{(x, y) \in E \mid(-x,-y) \in B\} .
$$

Subsequently, a multitude of $M M$ operators are derived from dilation and erosion such as morphological opening:

$$
\gamma_{g}(f)(x)=\delta_{\breve{g}}\left(\varepsilon_{g}(f)\right)(x)=((f \ominus g) \oplus g)(x), \forall x \in E_{f} \subseteq \mathbb{R}^{n}
$$

and morphological closing:

$$
\phi_{g}(f)(x)=\varepsilon_{\breve{g}}\left(\delta_{g}(f)\right)(x)=((f \oplus g) \ominus g)(x), \forall x \in E_{f} \subseteq \mathbb{R}^{n}
$$

and morphological gradient:

$$
\nabla_{g}(f)(x)=\delta_{g}(f)(x)-\varepsilon_{g}(f)(x)=\left((f \oplus g)(x)-(f \ominus g)(x), \forall x \in E_{f} \subseteq \mathbb{R}^{n}\right.
$$

where the structuring function $g: E_{g} \subseteq \mathbb{R}^{n} \rightarrow \mathbb{R}$ and the symmetric structuring function $\breve{g}(x)=g(-x), \forall x \in \mathbb{R}^{n}$, are the same semi-continuous functions defined above. In case of $2-D$ binary images morphological binary opening $(\circ)$ and binary closing $(\bullet)$ are respectively defined as:

$$
\begin{array}{r}
A \circ B=(A \ominus B) \oplus B^{S}=\left\{(x, y) \in B_{(u, v)} \wedge B_{(u, v)} \subseteq A\right\}= \\
=\bigcup_{(u, v) \in A}\left\{B_{(u, v)} \mid B_{(u, v)} \subseteq A\right\} \\
A \bullet B=(A \oplus B) \ominus B^{S}=\bigcap_{(u, v) \in A^{C}}\left\{B_{(u, v)}^{C} \mid B_{(u, v)} \subseteq A^{C}\right\}=\left(A^{C} \circ B^{S}\right)^{C}
\end{array}
$$

where $A$ is a 2-D binary image object and $B$ is a 2- $D$ structuring element defined on an Euclidian space, $E \in \mathbb{Z}^{2}$. $B^{S}$ denotes the symmetric structuring element $B . B_{(x, y)}$ is the translation of $B$ by vectors $(u, v) . A^{C}$ is the complement of $A$ relative to $E, A^{C}=\{(x, y) \mid(x, y) \in E \wedge(x, y) \notin A\}$. Besides morphological gradient $\left(\nabla_{B}\right)$ we can also define external contour $\left(\beta_{B}^{\text {ext }}\right)$ and internal contour $\left(\beta_{B}^{\text {int }}\right)$ for the $2-\mathrm{D}$ binary images: 


$$
\begin{gathered}
\nabla_{B}(A)=(A \oplus B)-(A \ominus B) \\
\beta_{B}^{e x t}(A)=(A \oplus B)-A ; \quad \beta_{B}^{\text {int }}(A)=A-(A \ominus B)
\end{gathered}
$$

Furthermore we can define a couple of more complex MM operators as the Top-Hat transforms. The White Top-Hat transform can detect and extract bright structures on non-uniform backgrounds and is defined as the difference between the input image and its opening by some structuring element:

$$
\begin{array}{r}
\tau_{g}^{W}(f)(x)=f(x)-\gamma_{g}(f)(x)=f(x)-\delta_{\breve{g}}\left(\varepsilon_{g}(f)\right)(x)= \\
=f(x)-((f \ominus g) \oplus g)(x), \forall x \in E_{f} \subseteq \mathbb{R}^{n}
\end{array}
$$

The Black Top-Hat is the dual transform and it can detect and extract dark structures on non-uniform backgrounds and is defined as the difference between the closing of an image by some structuring element and the same input image:

$$
\begin{array}{r}
\tau_{g}^{B}(f)(x)=\phi_{g}(f)(x)-f(x)=\varepsilon_{\breve{g}}\left(\delta_{g}(f)\right)(x)-f(x)= \\
=((f \oplus g) \ominus g)(x)-f(x), \forall x \in E_{f} \subseteq \mathbb{R}^{n}
\end{array}
$$

The skeleton of the input image $f(x)$ represents the union of all intersections of the differences between the erosion and its opening through variable sized structuring elements $g(x)$ (i.e. White Top-Hat transforms) [7]:

$$
S(f)=\bigcup_{\lambda \geq 0} \bigcap_{\mu \geq 0}\left[\varepsilon_{\lambda g}(f)(x)-\gamma_{\mu g}\left(\varepsilon_{\lambda g}(f)(x)\right)\right], \forall x \in E_{f} \subseteq \mathbb{R}^{n} .
$$

The Hit-or-Miss transform, locates either pixels from erosion $X-E_{1}$ (i.e. "hit") and from erosion $X^{c}-E_{2}^{c}$, (i.e. "miss") [7]:

$$
H M T_{E}(X)=\varepsilon_{E_{1}}(X) \bigcap \varepsilon_{E_{2}}\left(X^{C}\right)
$$

\section{Morphological signatures}

Morphological signatures can be obtained from a series of successive openings or closings. They can be extended from a pixel-related scale to an imagerelated scale (i.e. from a local definition using morphological profile operators to a global definition using pattern spectrum/granulometry operators) ([4], $[5])$.

The opening morphological signature series is defined as:

$$
\Gamma(f)(x)=\left\{\gamma_{k g}(f)(x) \mid k g=\delta_{S}^{k}(g)(x), \forall k \in\{0, \ldots, m\}, \forall x \in E_{f} \subseteq \mathbb{R}^{n}\right\}
$$


where $\gamma_{k g}(f)(x)$ is the opening by a variable sized structuring element $k g$ and $\delta_{S}^{k}(g)(x)$ is the $k$-times successive dilation of the structuring element $g$ by another very simple or elementary structuring element $S$. By definition $\gamma_{0}(f)(x)=f(x)$.

The dual closing morphological signature series is defined as:

$$
\Phi(f)(x)=\left\{\phi_{k g}(f)(x) \mid k g=\delta_{S}^{k}(g)(x), \forall k \in\{0, \ldots, m\}, \forall x \in E_{f} \subseteq \mathbb{R}^{n}\right\}
$$

Where $\phi_{k g}(f)(x)$ is the closing by a similar variable sized structuring element $k g$, as defined above, and $\delta_{S}^{k}(g)(x)$ is the same $k$-times successive dilation of the structuring element $g$ by another very simple or elementary structuring element $S$.

Subsequently, we can define the differential series computed from opening morphological signature series, which can provide more meaningful morphological information:

$$
\begin{array}{r}
\Delta \gamma(f)(x)=\left\{\gamma_{k g}(f)(x)-\gamma_{(k-1) g}(f)(x) \mid k g=\delta_{S}^{k}(g)(x),\right. \\
\left.\forall k \in\{0, \ldots, m\}, \forall x \in E_{f} \subseteq \mathbb{R}^{n}\right\}
\end{array}
$$

The dual differential series computed from closing morphological signature series can be defined as:

$$
\begin{array}{r}
\Delta \phi(f)(x)=\left\{\phi_{(k-1) g}(f)(x)-\phi_{k g}(f)(x) \mid k g=\delta_{S}^{k}(g)(x),\right. \\
\left.\forall k \in\{0, \ldots, m\}, \forall x \in E_{f} \subseteq \mathbb{R}^{n}\right\}
\end{array}
$$

Finally, morphological signatures are defined from these 4 series:

$$
\left\{\begin{array}{l}
\Gamma(f)(x) \\
\Phi(f)(x)) \\
\Delta \gamma(f)(x) \\
\Delta \phi(f)(x))
\end{array}\right.
$$

where $x \in E_{f} \subseteq \mathbb{R}^{n}$.

On a local pixel scale this set of 4 series is used to define differential morphological profile for a given pixel $x \in E_{f} \subseteq \mathbb{R}^{n}$ in image $f$. On a global image scale the pattern spectrum is built from the image series above gathering the image pixel values through the sum operation, i.e.

$$
\left\{\begin{array}{l}
\sum_{x \in E_{f}}(\Gamma(f)(x)+\Phi(f)(x)) \\
\sum_{x \in E_{f}}(\Delta \gamma(f)(x)+\Delta \phi(f)(x))
\end{array}\right.
$$

where $x \in E_{f} \subseteq \mathbb{R}^{n}$. 
The shape probability distribution function, involves a normalization by the initial image volume:

$$
\left\{\begin{array}{l}
\frac{\sum_{x \in E_{f}}(\Gamma(f)(x)+\Phi(f)(x))}{\sum_{x \in E_{f}}(f)(x)} \\
\frac{\sum_{x \in E_{f}}(\Delta \gamma(f)(x)+\Delta \phi(f)(x))}{\sum_{x \in E_{f}}(f)(x)}
\end{array}\right.
$$

where $x \in E_{f} \subseteq \mathbb{R}^{n}$.

\section{Morphological image indexing based on edge extrac- tion and homotopic skeleton}

One of the simplest morphological contour extraction methods is the morphological gradient: basically it consists of constructing an edge intensity map of the image as the difference between the local dilation and the local erosion at each image pixel (the word "local" being induced by the use of a structuring element with finite spatial support).

$$
\nabla_{B}(f)(x)=(f \oplus g)(x)-(f \ominus g)(x), \forall x \in E_{f} \subseteq \mathbb{R}^{2}
$$

Starting from the above definition of morphological gradient for the 2-D images we can compute the length of an object boundary or contour which is a meaningful local morphological signature. This can also be extended to a global image scale:

$$
L\left(\nabla_{B}(f)\right)=\sum_{x \in E_{f}} \nabla_{B}(f)(x)
$$

where $x \in E_{f} \subseteq \mathbb{R}^{2}$.

The first step is to generate an edge intensity map using the morphological gradient operator. The edge intensity map consists of values proportional to the local variation within each pixel neighborhood, as defined by the support of the structuring element. The binary edge map is obtained by thresholding the edge intensity map and selecting the pixels with a strong color (value) variations, measured by important values of the morphological gradient.

A typical edge extraction result by the described method is presented in figure 1.

Also, we can compute another important morphological signature as the length of the skeleton: 


$$
L(S(f))=\sum_{x \in E_{f}} \bigcup_{\lambda \geq 0}\left[\varepsilon_{\lambda g}(f)(x)-\gamma_{\lambda g}\left(\varepsilon_{\lambda g}(f)(x)\right)\right], \forall x \in E_{f} \subseteq \mathbb{R}^{2}
$$

The length of the skeleton was used as a very meaningful morphological signature in the experiment concerning labelling and classification of color image objects as shown in Figure 2.

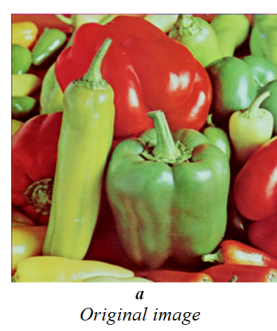

Original image

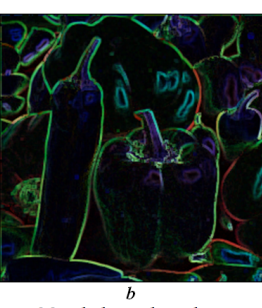

Morphological gradient

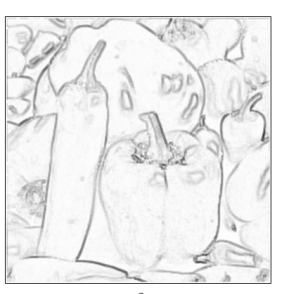

Edge-intensity map extracted by gradient form the original image.

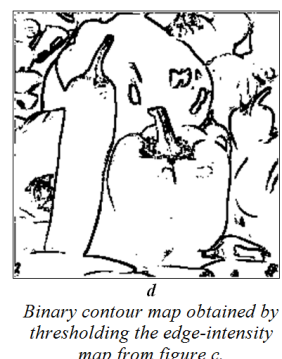

map from figure $c$.

Figure 1. Morphological edge extraction for color image

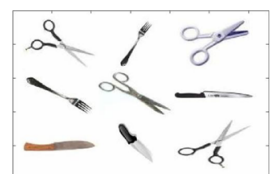

so) Original image

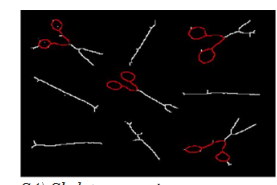

S4) Skeleton pruning

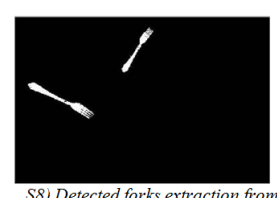

S8) Detected forks
the B\&W image

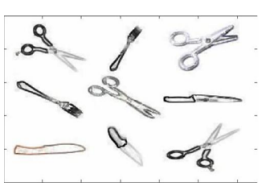

SI) Morphological gradient
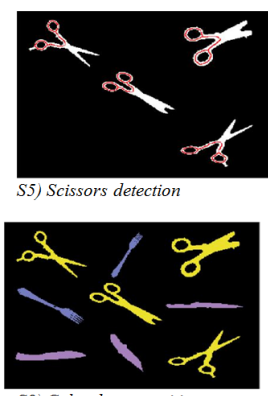

Color decomposition

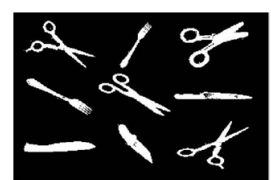

S2) Binary image conversion

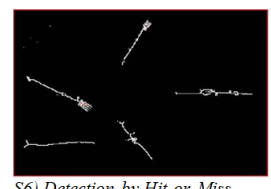

S6) Detection by Hit-or-Miss

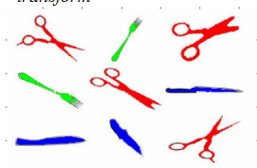

S10) Color labeling the inputcolo image

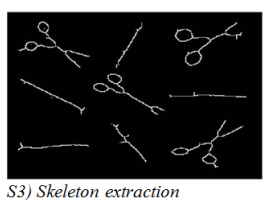

3) Skeleton extraction

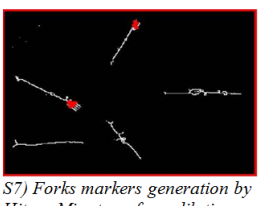

S7) Forks markers generation by
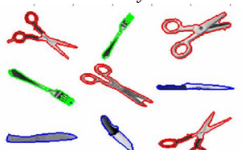
S1 1) Final color classification of harp-edged objects

Figure 2. Labelling and classification of sharp-edged objects in a color image

Acknowledgement: The publication of this article was supported by the grant PN-II-ID-WE-2012-4-169 of the Workshop "A new approach in theoretical and applied methods in algebra and analysis". 


\section{References}

[1] J. Angulo, J. Serra, "Color segmentation by ordered mergings", Proc. of IEEE International Conference on Image Processing (ICIP'03), IEEE, Vol. 2, Barcelona, Spain, Sept. 2003, p. 125-128.

[2] J. Angulo, J. Serra, "Mathematical morphology in color spaces applied to the analysis of cartographic images", Proc. of IEEE International Conference on Image Processing (ICIP'07), 2007.

[3] Aptoula, E., Lefèvre, S.: "A comparative study on multivariate mathematical morphology", Pattern Recognition (2007).

[4] Lefèvre, S., Weber, J., Sheeren, D.: "Automatic building extraction in vhr images using advanced morphological operators", IEEE/ISPRS Joint Workshop on Remote Sensing and Data Fusion over Urban Areas, Paris, France (2007).

[5] Lefèvre, S., "Extending Morphological Signature for Visual Pattern Recognition", Pattern Recognition (2009).

[6] J. Serra, "Image Analysis and Mathematical Morphology". Vol I, and "Image Analysis and Mathematical Morphology.Theoretical Advances". Vol II: London: Academic Press, 1982,1988.

[7] J. Serra, "Image Analysis and Mathematical Morphology" Academic Press, London, 1982.

[8] P. Soille, "Morphological Image Analysis", Springer-Verlag, Berlin, Heidelberg, New York, 1999.

[9] E. Zaharescu, M. Zamfir, C.Vertan: "Color Morphology-like Operators based on Color Geometric Shape Characteristics", Proc. of International Symposium on Signals Circuits and Systems SCS 2003, Iaşi, Romania, 2003.

[10] E. Zaharescu, "Morphological Algorithm for Color Objects Classification", IEEE Computer Society Proceedings of the Fourth Balkan Conference in Informatics, 17-19 September 2009, Thessaloniki, Greece, pag.152156, Library of Congress No. 2009934322, (ISBN 978-0-7695-3783-2).

"Ovidius" University of Constanta,

Faculty of Mathematics and Informatics,

124 Mamaia Blvd., 900527 Constanta, Romania,

e-mail: ezaharescu@univ-ovidius.ro 